\title{
$J$-Self-Adjoint Projections in Krein Spaces
}

\author{
Xiao-Ming $\mathrm{Xu} \mathbb{D}^{1}$ and Yile Zhao ${ }^{2}$ \\ ${ }^{1}$ School of Science, Shanghai Institute of Technology, Shanghai 201418, China \\ ${ }^{2}$ Department of Mathematics, Hangzhou Normal University, Hangzhou 310036, China \\ Correspondence should be addressed to Xiao-Ming Xu; xuxiaoming2620@aliyun.com
}

Received 9 October 2019; Revised 12 February 2020; Accepted 22 February 2020; Published 16 March 2020

Academic Editor: Giuseppe Marino

Copyright (C) 2020 Xiao-Ming $\mathrm{Xu}$ and Yile Zhao. This is an open access article distributed under the Creative Commons Attribution License, which permits unrestricted use, distribution, and reproduction in any medium, provided the original work is properly cited.

Let $\mathscr{H}$ be a Krein space with fundamental symmetry $J$. Starting with a canonical block-operator matrix representation of $J$, we study the regular subspaces of $\mathscr{H}$. We also present block-operator matrix representations of the J-self-adjoint projections for the regular subspaces of $\mathscr{H}$, as well as for the regular complements of the isotropic part in a pseudo-regular subspace of $\mathscr{H}$.

\section{Introduction}

Throughout this paper, let $\mathscr{H}$ be a separable complex Hilbert space with the inner product $\langle\cdot, \cdot\rangle$ and let $\mathscr{B}(\mathscr{H})$ be the algebra of all bounded linear operators on $\mathscr{H}$. A contraction in $\mathscr{B}(\mathscr{H})$ is an operator $Q$ in $\mathscr{B}(\mathscr{H})$ such that $\|Q\| \leq 1$. For an operator $T \in \mathscr{B}(\mathscr{H}), T^{*}, \sigma(T), \sigma_{P}(T), \mathscr{R}(T)$, and $\mathscr{N}(T)$ denote the adjoint, the spectrum, the point spectrum, the range, and the null space of $T$, respectively. An operator $T$ in $\mathscr{B}(\mathscr{H})$ is said to be self-adjoint if $T=T^{*}$.

An operator $J$ in $\mathscr{B}(\mathscr{H})$ is said to be a symmetry (or selfadjoint involution) if $J=J^{*}=J^{1}$. If the symmetry $J$ is nonscalar, then

$$
[x, y]=\langle J x, y\rangle
$$

which defines an indefinite inner product on $\mathscr{H}$, and $(\mathscr{H}, J)$ is called a Krein space $([1-3])$.

For $T \in \mathscr{B}(\mathscr{H})$, the $J$-adjoint operator of $T$ is the unique operator $T^{\sharp}$ in $\mathscr{B}(\mathscr{H})$ satisfying

$$
[T x, y]=\left[x, T^{\sharp} y\right],
$$

for all $x, y \in \mathscr{H}$. It is easy to see that $T^{\sharp}=J T^{*} J$.

Definition 1. If $T \in \mathscr{B}(\mathscr{H})$, then (a) $T$ is $J$-normal if $T T^{\sharp}=T^{\sharp} T$; (b) $T$ is $J$-self-adjoint if $T^{\sharp}=T$; (c) $T$ is $J$-positive if $J T \geq 0$; (d) $T$ is $J$-negative if $J T \leq 0$.
An idempotent in $\mathscr{B}(\mathscr{H})$ is called a projection. A projection is normal if and only if it is self-adjoint. However, there exist $J$-normal projections which are not $J$-self-adjoint (see [4]).

For a subspace $\mathcal{S}$ of the Krein space $\mathscr{H}, \mathcal{S}^{[\perp]}$ denotes the $J$-orthocomplement of $\mathcal{S}$ in $\mathscr{H}$, that is,

$$
\mathcal{S}^{[\perp]}=\{h \in \mathscr{H}:[h, s]=0 \text { for all } s \text { in } \mathcal{S}\} .
$$

It is obvious that $\mathcal{\delta}^{[\perp]}$ equals the usual orthocomplement $(J \mathcal{S})^{\perp}$. Let $\mathcal{S}^{0}:=S \cap \mathcal{S}^{[\perp]}$ be the isotropic part of $\mathcal{S}$. If $\mathcal{S}^{0}=\{0\}$, then $\mathcal{S}$ is said to be $J$-nondegenerate. Otherwise, $\mathcal{S}$ is said to be $J$-degenerate.

Definition 2. If $\mathcal{S}$ is a subspace of the Krein space $\mathscr{H}$, then (a) $\mathcal{S}$ is positive if $P_{\mathcal{S}} J P_{\mathcal{S}} \geq 0$, where $P_{\mathcal{S}}$ is the orthogonal projection of $\mathscr{H}$ onto $\mathcal{S}$; (b) $\mathcal{S}$ is uniformly positive if $P_{\mathcal{\delta}} J P_{\mathcal{S}} \geq \varepsilon P_{\mathcal{\delta}}$ for some $\epsilon>0$; (c) $\mathcal{S}$ is regular if $\mathscr{H}=\mathcal{S} \dot{+} \mathcal{S}^{[\perp]}$; (d) $\mathcal{S}$ is pseudoregular if $\mathcal{S}$ and the algebraic sum $\mathcal{S}+\mathcal{S}^{[\perp]}$ are closed.

It is well known that a subspace $\delta$ of $\mathscr{H}$ is regular if and only if it is the range of a (unique) $J$-self-adjoint projection in $\mathscr{B}(\mathscr{H})$ (see [2]). Therefore, there is a one-to-one correspondence between regular subspaces of $\mathscr{H}$ and $J$-self-adjoint projections in $\mathscr{B}(\mathscr{H})$. It is also proved in [5] that a closed subspace $\delta$ is regular if and only if $\left(P_{\mathcal{\delta}} J P_{\mathcal{\delta}}\right)^{2} \geq \varepsilon P_{\mathcal{\delta}}$ for some $\epsilon>0$. In consequence, a closed subspace $\delta$ is uniformly positive if and only if it is regular and positive, and 
due to Proposition 4 in [6], this is the case if and only if it is a regular subspace with a $J$-positive projection.

Pseudoregular subspaces are important since they enable to generalize some Pontryagin space arguments to general Krein space (see [7]). Pseudoregular subspaces and its properties have been studied extensively by many authors (see $[4,7,8]$ ). In [4], the authors proved that a closed subspace $\mathcal{S}$ of $\mathscr{H}$ is pseudoregular if and only if it is the range of a $J$-normal projection in $\mathscr{B}(\mathscr{H})$. They also showed in the same paper that a pseudoregular subspace $\mathcal{S}$ admits infinitely many $J$-normal projections onto it, unless $\mathcal{S}$ is regular. In [8], Giribet et al. gave a block-operator matrix representation of the fundamental symmetry $J$ depending on a pseudoregular subspace $\mathcal{S}$ of $\mathscr{H}$, and from here on, they characterized the $J$-self-adjoint projections for the regular complements of $\mathcal{S}^{0}$ in $\mathcal{S}$.

In this paper, we give a new block-operator matrix representation of the fundamental symmetry $J$ related to a closed subspace $\mathcal{S}$ of $\mathscr{H}$. This offers an improvement over the result in [8], since we do not need to impose the assumption of the pseudoregularity of $\mathcal{S}$. We also study the $J$ self-adjoint projections for the regular subspaces of $\mathscr{H}$, as well as for the regular complements of the isotropic part in a pseudoregular subspace of $\mathscr{H}$.

The paper is organized as follows. In Section 2, we give a block-operator matrix representation of the fundamental symmetry $J$. Therein, we also characterize the regular subspaces of $\mathscr{H}$ and present a block-operator matrix representation of the $J$-self-adjoint projections for the regular subspaces of $\mathscr{H}$. In Section 3, we study the pseudoregular subspaces of $\mathscr{H}$. If $\mathcal{S}$ is a pseudoregular subspace of $\mathscr{H}$ and $\mathscr{L}$ is a regular complement of $\mathcal{S}^{0}$ in $\mathcal{S}$, we give a blockoperator matrix representation of the J-self-adjoint projection onto $\mathscr{L}$.

\section{J-Self-Adjoint Projections for the Regular Subspaces}

Let $\mathscr{H}$ be a Krein space with fundamental symmetry $J$. Then, $J^{+}:=((I+J) / 2)$ and $J^{-}:=((I-J) / 2)$ are mutually annihilating orthogonal projections. Denote $\mathscr{H}_{+}=\mathscr{R}\left(J^{+}\right)$and $\mathscr{H}_{-}=\mathscr{R}\left(J^{-}\right)$. We have fundamental decomposition $\mathscr{H}=\mathscr{H}_{+} \oplus \mathscr{H}_{-}$.

Let $\mathcal{S}$ be a closed subspace of the Krein space $\mathscr{H}$, and let $\mathcal{S}^{0}$ be its isotropic part. Denote $\mathscr{H}_{1}=\mathcal{S} \cap \mathscr{H}_{+}, \mathscr{H}_{2}=\mathcal{S} \cap \mathscr{H}_{-}$, $\mathscr{H}_{3}=\mathcal{S}^{0}, \mathscr{H}_{4}=\mathcal{S} \ominus\left(\mathscr{H}_{1} \oplus \mathscr{H}_{2} \oplus \mathscr{H}_{3}\right), \mathscr{H}_{5}=\mathcal{S}^{\perp} \ominus\left(J\left(\mathcal{S}^{0}\right) \oplus\right.$ $\left.\left(\mathcal{S}^{\perp} \cap \mathscr{H}_{+}\right) \oplus\left(\mathcal{S}^{\perp} \cap \mathscr{H}_{-}\right)\right), \mathscr{H}_{6}=J\left(\mathcal{S}^{0}\right), \mathscr{H}_{7}=\mathcal{S}^{\perp} \cap \mathscr{H}_{+}$, and $\mathscr{H}_{8}=\mathcal{S}^{\perp} \cap \mathscr{H}_{-}$. It is easy to check that $\mathscr{H}_{i}, 1 \leq i \leq 8$, are pairwise orthogonal subspaces of $\mathscr{H}$. The operators in this paper are frequently treated as block-operator matrices with respect to the space decomposition:

$$
\mathscr{H}=\mathscr{H}_{1} \oplus \mathscr{H}_{2} \oplus\left(\mathscr{H}_{3} \oplus \mathscr{H}_{4} \oplus \mathscr{H}_{5} \oplus \mathscr{H}_{6}\right) \oplus \mathscr{H}_{7} \oplus \mathscr{H}_{8} .
$$

For a pseudoregular subspace $\delta$ of the Krein space $\mathscr{H}$, a block-operator matrix representation of the fundamental symmetry $J$ was obtained with the space decomposition $\mathscr{H}=$ $\mathcal{S}^{0} \oplus\left(\mathcal{S} \ominus \mathcal{S}^{0}\right) \oplus\left(\mathcal{S}^{\perp} \ominus J\left(\mathcal{S}^{0}\right)\right) \oplus J\left(\mathcal{S}^{0}\right)$ in [8]. We continue the study of the block-operator matrix representation of the fundamental symmetry $J$, but we do not impose the assumption of the pseudoregularity of the subspace $\mathcal{S}$.

Theorem 1. Let $\mathscr{H}$ be a Krein space with fundamental symmetry J, and let $\mathcal{S}$ be a closed subspace of $\mathscr{H}$. Then, J has the operator matrix representation:

$$
J=I_{1} \oplus-I_{2} \oplus\left(\begin{array}{cccc}
0 & 0 & 0 & U \\
0 & Q & \left(I_{4}-Q^{2}\right)^{1 / 2} V & 0 \\
0 & V^{*}\left(I_{4}-Q^{2}\right)^{1 / 2} & -V^{*} Q V & 0 \\
U^{*} & 0 & 0 & 0
\end{array}\right)
$$$$
\oplus I_{7} \oplus-I_{8}
$$

with respect to space decomposition (4), where $I_{i}$ is the identity operator on the corresponding space $\mathscr{H}_{i}, i=1,2,4,7,8, U$ is an isometric isomorphism from $\mathscr{H}_{6}$ onto $\mathscr{H}_{3}, V$ is an isometric isomorphism from $\mathscr{H}_{5}$ onto $\mathscr{H}_{4}$, and $Q$ is a self-adjoint contraction on $\mathscr{H}_{4}$ with $0, \pm 1 \notin \sigma_{P}(Q)$.

Proof. It is clear that $\mathscr{H}_{1}$ and $\mathscr{H}_{7}$ are subspaces of $\mathscr{H}^{+}$and $\mathscr{H}_{2}$ and $\mathscr{H}_{8}$ are subspaces of $\mathscr{H}^{-}$. So $\left.J\right|_{\mathscr{H}^{+}}=\left.I\right|_{\mathscr{H}^{+}}$implies $\left.J\right|_{\mathscr{H}_{1}}=\left.I\right|_{\mathscr{H}_{1}}$ and $\left.J\right|_{\mathscr{H}_{7}}=\left.I\right|_{\mathscr{H}_{7}}$ and $\left.J\right|_{\mathscr{H}^{-}}=-\left.I\right|_{\mathscr{H}^{-}}$implies $\left.J\right|_{\mathscr{H}_{2}}=$ $-\left.I\right|_{\mathscr{H}_{2}}$ and $\left.J\right|_{\mathscr{H}_{8}}=-\left.I\right|_{\mathscr{H}_{8}}$. Since $J\left(\mathscr{H}_{3}\right)=\mathscr{H}_{6}$, we have $\left.P_{\mathscr{H}_{i}} J\right|_{\mathscr{H}_{3}}=0$ for $i \neq 6$. Moreover, since $J\left(\mathscr{H}_{6}\right)=J\left(J\left(\mathscr{H}_{3}\right)\right)=$ $J^{2}\left(\mathscr{H}_{3}\right)^{3}=I\left(\mathscr{H}_{3}\right)=\mathscr{H}_{3}$, we get $P_{\mathscr{H}_{i}} J_{\mathscr{H}_{6}}=0$ for $i \neq 3$. Noting that $J$ is self-adjoint, then $J$ has the operator matrix representation:

$$
J=I_{1} \oplus-I_{2} \oplus\left(\begin{array}{cccc}
0 & 0 & 0 & J_{36} \\
0 & J_{44} & J_{45} & 0 \\
0 & J_{45}^{*} & J_{55} & 0 \\
J_{36}^{*} & 0 & 0 & 0
\end{array}\right) \oplus I_{7} \oplus-I_{8}
$$

with respect to space decomposition (4), where $I_{i}$ is the identity operator on the corresponding space $\mathscr{H}_{i}$, $i=1,2,7,8, J_{36} \in \mathscr{B}\left(\mathscr{H}_{6}, \mathscr{H}_{3}\right), J_{45} \in \mathscr{B}\left(\mathscr{H}_{5}, \mathscr{H}_{4}\right)$, and $J_{44}$ and $J_{55}$ are self-adjoint contractions on $\mathscr{H}_{4}$ and $\mathscr{H}_{5}$, respectively:

Let $J_{36}=U$. Since $J^{2}=I$, it is easy to see that $U U^{*}=I_{3}$ and $U^{*} U=I_{6}$. Thus, $U$ is an isometric isomorphism from $\mathscr{H}_{6}$ onto $\mathscr{H}_{3}$.

Let $J_{44}=Q$. If $\xi \in \mathcal{N}\left(I_{4}-Q\right)$ and $x=(0,0,0, \xi, 0,0,0,0)^{T}$, then

$$
\begin{aligned}
J x & =\left(0,0,0, Q \xi, J_{45}^{*} \xi, 0,0,0\right)^{T} \\
& =\left(0,0,0, \xi, J_{45}^{*} \xi, 0,0,0\right)^{T} .
\end{aligned}
$$

Since $\|J\|=1, J_{45}^{*} \xi=0$ and $J x=x$. It follows that $x \in \mathscr{H}_{+}$, and hence, $x \in \mathcal{S} \cap \mathscr{H}_{+}=\mathscr{H}_{1}$. This implies $\xi=0$. Thus, $1 \notin \sigma_{P}(Q)$. Analogously, $-1 \notin \sigma_{P}(Q)$ and $\pm 1 \notin \sigma_{P}\left(J_{55}\right)$.

Moreover, if $\xi \in \mathcal{N}(Q)$ and $x=(0,0,0, \xi, 0,0,0,0)^{T}$, then

$$
[x, y]=\langle J x, y\rangle=\langle Q \xi, y\rangle+\left\langle J_{45}^{*} \xi, y\right\rangle=\langle Q \xi, y\rangle=0,
$$

for all $y \in \mathcal{S}$. It follows that $x \in \mathcal{S}^{[\perp]}$, and hence, $x \in \mathcal{S} \cap \mathcal{S}^{[\perp]}=\mathcal{S}^{0}=\mathscr{H}_{3}$. So $\xi=0$, and hence, $0 \notin \sigma_{P}(Q)$. 
Let $J^{\prime}=\left.P_{\mathscr{H}_{4} \oplus \mathscr{H}_{5}} J\right|_{\mathscr{H}_{4} \oplus \mathscr{H}_{5}}$, that is,

$$
J^{\prime}=\left(\begin{array}{cc}
Q & J_{45} \\
J_{45}^{*} & J_{55}
\end{array}\right): \mathscr{H}_{4} \oplus \mathscr{H}_{5} \longrightarrow \mathscr{H}_{4} \oplus \mathscr{H}_{5} .
$$

Then, $J^{\prime}$ is a symmetry. So we have

$$
J^{\prime^{+}}=\frac{1}{2}\left(I_{4} \oplus I_{5}+J^{\prime}\right)=\frac{1}{2}\left(\begin{array}{cc}
I_{4}+Q & J_{45} \\
J_{45}^{*} & I_{5}+J_{55}
\end{array}\right) \geq 0,
$$

and by Proposition 5 in [9], there exists a contraction $V$ from $\mathscr{H}_{5}$ into $\mathscr{H}_{4}$ such that

$$
J_{45}=\left(I_{4}+Q\right)^{1 / 2} V\left(I_{5}+J_{55}\right)^{1 / 2} .
$$
implies

Then, by a direct calculation, equation $\left(J^{\prime^{+}}\right)^{2}=J^{\prime^{+}}$

$$
\left\{\begin{array}{l}
\left(I_{4}+Q\right)^{2}+\left(I_{4}+Q\right)^{1 / 2} V\left(I_{5}+J_{55}\right) V^{*}\left(I_{4}+Q\right)^{1 / 2}=2\left(I_{4}+Q\right), \\
\left(I_{4}+Q\right)^{3 / 2} V\left(I_{5}+J_{55}\right)^{1 / 2}+\left(I_{4}+Q\right)^{1 / 2} V\left(I_{5}+J_{55}\right)^{3 / 2}=2\left(I_{4}+Q\right)^{1 / 2} V\left(I_{5}+J_{55}\right)^{1 / 2}, \\
\left(I_{5}+J_{55}\right)^{1 / 2} V^{*}\left(I_{4}+Q\right) V\left(I_{5}+J_{55}\right)^{1 / 2}+\left(I_{5}+J_{55}\right)^{2}=2\left(I_{5}+J_{55}\right) .
\end{array}\right.
$$

Noting that $-1 \notin \sigma_{P}(Q)$ and $-1 \notin \sigma_{P}\left(J_{55}\right)$, it follows that

$$
\left\{\begin{array}{l}
\text { (i) } I_{4}-Q=V\left(I_{5}+J_{55}\right) V^{*}, \\
\text { (ii) }-Q V=V J_{55} \\
\text { (iii) } V^{*}\left(I_{4}+Q\right) V=I_{5}-J_{55} .
\end{array}\right.
$$

By (11) and (ii) of (13), we obtain

$$
\begin{aligned}
J_{45} & =\left(I_{4}+Q\right)^{1 / 2} V\left(I_{5}+J_{55}\right)^{1 / 2}=\left(I_{4}+Q\right)^{1 / 2}\left(I_{4}-Q\right)^{1 / 2} V \\
& =\left(I_{4}-Q^{2}\right)^{1 / 2} V .
\end{aligned}
$$

By (i) and (ii) of (13), $\left(I_{4}-Q\right)\left(V V^{*}-I_{4}\right)=0$, and since $I_{4}-Q$ is injective, $V V^{*}=I_{4}$. By (ii) and (iii) of (13), $\left(V^{*} V-I_{5}\right)$ $\left(I_{5}-J_{55}\right)=0$, and since $I_{5}-J_{55}$ is a self-adjoint operator with dense range in $\mathscr{H}_{5}, V^{*} V=I_{5}$. Thus, $V$ is an isometric isomorphism from $\mathscr{H}_{5}$ onto $\mathscr{H}_{4}$, and by (ii) of (13) again,

$$
J_{55}=-V^{*} Q V .
$$

Now we see that

$J^{\prime}=\left(\begin{array}{cc}Q & \left(I_{4}-Q^{2}\right)^{1 / 2} V \\ V^{*}\left(I_{4}-Q^{2}\right)^{1 / 2} & -V^{*} Q V\end{array}\right): \mathscr{H}_{4} \oplus \mathscr{H}_{5} \longrightarrow \mathscr{H}_{4} \oplus \mathscr{H}_{5}$,

and $J$ has the asserted operator matrix.

Lemma 1 (see [5]). A closed subspace $\delta$ is regular if and only if $\left(P_{\delta} J P_{\delta}\right)^{2} \geq \epsilon P_{\delta}$ for some $\epsilon>0$. In this case, the J-selfadjoint projection onto $\delta$ is determined as $\left(P_{\delta} J P_{\delta}\right)^{\dagger} J$, where $\left(P_{\delta} J P_{\delta}\right)^{\dagger}$ stands for the Moore-Penrose inverse of $P_{\delta} J P_{\delta}$.

Theorem 2. Let $\mathscr{H}$ be a Krein space with fundamental symmetry J, and let $\delta$ be a closed subspace of $\mathscr{H}$. Write J in (5) with respect to space decomposition (4). Then, $\mathcal{S}$ is regular if and only if $\mathscr{H}_{3}=\{0\}$ and $0 \notin \sigma(Q)$. In this case, $\mathscr{H}_{6}=\{0\}$ and with respect to the space decomposition $\mathscr{H}=\mathscr{H}_{1} \oplus \mathscr{H}_{2} \oplus$ $\left(\mathscr{H}_{4} \oplus \mathscr{H}_{5}\right) \oplus \mathscr{H}_{7} \oplus \mathscr{H}_{8}$, J and the J-self-adjoint projection $E$ onto $\mathcal{S}$ have operator matrix representations:
$J=I_{1} \oplus-I_{2} \oplus\left(\begin{array}{cc}Q & \left(I_{4}-Q^{2}\right)^{1 / 2} V \\ V^{*}\left(I_{4}-Q^{2}\right)^{1 / 2} & -V^{*} Q V\end{array}\right) \oplus I_{7} \oplus-I_{8}$

and

$$
E=I_{1} \oplus I_{2} \oplus\left(\begin{array}{cc}
I_{4} & Q^{-1}\left(I_{4}-Q^{2}\right)^{1 / 2} V \\
0 & 0
\end{array}\right) \oplus 0 \oplus 0,
$$

respectively.

Proof. It is clear that $P_{\mathcal{\delta}}$ has the operator matrix representation:

$$
P_{\mathcal{S}}=I_{1} \oplus I_{2} \oplus I_{3} \oplus I_{4} \oplus 0 \oplus 0 \oplus 0 \oplus 0,
$$

with respect to space decomposition (4), and by a direct calculation,

$$
P_{\delta} J P_{\mathcal{S}}=I_{1} \oplus-I_{2} \oplus 0 \oplus Q \oplus 0 \oplus 0 \oplus 0 \oplus 0 .
$$

Then, by Lemma $1, \mathcal{S}$ is regular if and only if $\mathscr{H}_{3}=\{0\}$ and $Q^{2} \geq \epsilon I_{4}$ for some $\epsilon>0$, and since $Q$ is self-adjoint, this is the case if and only if $\mathscr{H}_{3}=\{0\}$ and $0 \notin \sigma(Q)$.

If $\mathcal{S}$ is regular, then $\mathscr{H}_{6}=J\left(\mathscr{H}_{3}\right)=\{0\}$, and hence, $\mathscr{H}=\mathscr{H}_{1} \oplus \mathscr{H}_{2} \oplus \mathscr{H}_{4} \oplus \mathscr{H}_{5} \oplus \mathscr{H}_{7} \oplus \mathscr{H}_{8}$. By Theorem 1, $J$ has the operator matrix representation given in (17), and since $P_{\mathcal{S}}=I_{1} \oplus I_{2} \oplus I_{4} \oplus 0 \oplus 0 \oplus 0$ with respect to $\mathscr{H}=\mathscr{H}_{1} \oplus \mathscr{H}_{2} \oplus$ $\mathscr{H}_{4} \oplus \mathscr{H}_{5} \oplus \mathscr{H}_{7} \oplus \mathscr{H}_{8}$, we have

$$
\begin{aligned}
\left(P_{\mathcal{S}} J P_{\mathcal{S}}\right)^{\dagger} & =\left(I_{1} \oplus-I_{2} \oplus Q \oplus 0 \oplus 0 \oplus 0\right)^{\dagger} \\
& =I_{1} \oplus-I_{2} \oplus Q^{-1} \oplus 0 \oplus 0 \oplus 0 .
\end{aligned}
$$

Then, by Lemma 1, we get

$E=\left(P_{\mathcal{\delta}} J P_{\mathcal{S}}\right)^{\dagger} J=I_{1} \oplus I_{2} \oplus\left(\begin{array}{cc}I_{4} & Q^{-1}\left(I_{4}-Q^{2}\right)^{1 / 2} V \\ 0 & 0\end{array}\right) \oplus 0 \oplus 0$,

with respect to $\mathscr{H}=\mathscr{H}_{1} \oplus \mathscr{H}_{2} \oplus\left(\mathscr{H}_{4} \oplus \mathscr{H}_{5}\right) \oplus \mathscr{H}_{7} \oplus \mathscr{H}_{8}$.

Recall that a closed subspace $\mathcal{S}$ of $\mathscr{H}$ is uniformly positive if and only if it is a regular subspace with a $J$-positive 
projection in $\mathscr{B}(\mathscr{H})$. We give a characterization of the uniformly positive subspaces of the Krein space $\mathscr{H}$.

Corollary 1. Let $\mathscr{H}$ be a Krein space with fundamental symmetry J, and let $\mathcal{S}$ be a closed subspace of $\mathscr{H}$. Write J in (5) with respect to space decomposition (4). Then, $\mathcal{S}$ is uniformly positive if and only if $\mathscr{H}_{2}=\{0\}, \mathscr{H}_{3}=\{0\}$, and $Q$ is a positive operator in $\mathscr{B}\left(\mathscr{H}_{4}\right)$ with $0 \notin \sigma(Q)$. In this case, the blockoperator matrix representation of the J-positive projection $E$ onto $\mathcal{S}$ is given by

$$
E=I_{1} \oplus\left(\begin{array}{cc}
I_{4} & Q^{-1}\left(I_{4}-Q^{2}\right)^{1 / 2} V \\
0 & 0
\end{array}\right) \oplus 0 \oplus 0,
$$

with respect to the space decomposition $\mathscr{H}=\mathscr{H}_{1} \oplus\left(\mathscr{H}_{4} \oplus \mathscr{H}_{5}\right) \oplus \mathscr{H}_{7} \oplus \mathscr{H}_{8}$.

Proof (Necessity). Suppose that the closed subspace $\mathcal{S}$ is uniformly positive. Then, $\mathcal{S}$ is regular, and suppose that $E$ is the $J$-self-adjoint projection onto $\mathcal{S}$. By Theorem $2, \mathscr{H}_{3}=\{0\}$, $0 \notin \sigma(Q)$, and with respect to $\mathscr{H}=\mathscr{H}_{1} \oplus \mathscr{H}_{2} \oplus\left(\mathscr{H}_{4} \oplus \mathscr{H}_{5}\right) \oplus$ $\mathscr{H}_{7} \oplus \mathscr{H}_{8}$, J and $E$ have operator matrix representations (17) and (18), respectively. By a direct calculation, we have

$$
J E=I_{1} \oplus-I_{2} \oplus\left(\begin{array}{lc}
Q & \left(I_{4}-Q^{2}\right)^{1 / 2} V \\
V^{*}\left(I_{4}-Q^{2}\right)^{1 / 2} & V^{*}\left(I_{4}-Q^{2}\right) Q^{-1} V
\end{array}\right) \oplus 0 \oplus 0,
$$

and since $J E \geq 0$, we see that $\mathscr{H}_{2}=\{0\}$ and $Q \geq 0$.

Sufficiency. Suppose that $\mathscr{H}_{2}=\{0\}, \mathscr{H}_{3}=\{0\}$, and $Q$ is a positive operator in $\mathscr{B}\left(\mathscr{H}_{4}\right)$ with $0 \notin \sigma(Q)$. Then, by Theorem $1, J$ has the operator matrix representation:

$$
J=I_{1} \oplus\left(\begin{array}{lc}
Q & \left(I_{4}-Q^{2}\right)^{1 / 2} V \\
V^{*}\left(I_{4}-Q^{2}\right)^{1 / 2} & -V^{*} Q V
\end{array}\right) \oplus I_{7} \oplus-I_{8},
$$

with respect to $\mathscr{H}=\mathscr{H}_{1} \oplus\left(\mathscr{H}_{4} \oplus \mathscr{H}_{5}\right) \oplus \mathscr{H}_{7} \oplus \mathscr{H}_{8}$, and by Theorem $2, \mathcal{S}$ is regular and the $J$-self-adjoint projection $E$ onto $\mathcal{S}$ has the operator matrix representation:

$$
E=I_{1} \oplus\left(\begin{array}{cc}
I_{4} & Q^{-1}\left(I_{4}-Q^{2}\right)^{1 / 2} V \\
0 & 0
\end{array}\right) \oplus 0 \oplus 0
$$

with respect to $\mathscr{H}=\mathscr{H}_{1} \oplus\left(\mathscr{H}_{4} \oplus \mathscr{H}_{5}\right) \oplus \mathscr{H}_{7} \oplus \mathscr{H}_{8}$. It follows that

$$
\begin{aligned}
J E= & I_{1} \oplus\left(\begin{array}{lr}
Q & \left(I_{4}-Q^{2}\right)^{1 / 2} V \\
V^{*}\left(I_{4}-Q^{2}\right)^{1 / 2} & V^{*}\left(I_{4}-Q^{2}\right) Q^{-1} V
\end{array}\right) \oplus 0 \oplus 0 \\
= & I_{1} \oplus\left(\begin{array}{lr}
Q^{1 / 2} & 0 \\
V^{*}\left(I_{4}-Q^{2}\right)^{1 / 2} Q^{-(1 / 2)} & 0
\end{array}\right) \\
& \cdot\left(\begin{array}{cc}
Q^{1 / 2} & \left(I_{4}-Q^{2}\right)^{1 / 2} Q^{-(1 / 2)} V \\
0 & 0
\end{array}\right) \oplus 0 \oplus 0 \\
\geq 0 &
\end{aligned}
$$

and hence, $\mathcal{S}$ is uniformly positive.

Remark 1. Let $\mathcal{S}$ be a subspace of the Krein space $\mathscr{H}$. Then, $\mathcal{S}$ is said to be negative (resp., uniformly negative) if $P_{\mathcal{S}} J P_{\mathcal{S}} \leq 0$ (resp., $P_{\mathcal{\delta}} J P_{\mathcal{S}} \leq-\varepsilon P_{\mathcal{S}}$ for some $\epsilon>0$ ). If more $\mathcal{S}$ is closed, then $\mathcal{S}$ is uniformly negative if and only if it is regular and negative, or equivalently, if and only if it is a regular subspace with a $J$-negative projection. Arguing as in Corollary 1, we can also give a characterization of the uniformly negative subspaces of the Krein space $\mathscr{H}$. With the notation as in Corollary 1, a closed subspace $\mathcal{S}$ is uniformly negative if and only if $\mathscr{H}_{1}=\{0\}, \mathscr{H}_{3}=\{0\}$, and $-Q$ is a positive operator in $\mathscr{B}\left(\mathscr{H}_{4}\right)$ with $0 \notin \sigma(Q)$. In this case, the block matrix representation of the $J$-negative projection $F$ onto $\mathcal{S}$ is given by

$$
F=I_{2} \oplus\left(\begin{array}{cr}
I_{4} & Q^{-1}\left(I_{4}-Q^{2}\right)^{1 / 2} V \\
0 & 0
\end{array}\right) \oplus 0 \oplus 0,
$$

with respect to the space decomposition $\mathscr{H}=\mathscr{H}_{2} \oplus\left(\mathscr{H}_{4} \oplus \mathscr{H}_{5}\right) \oplus \mathscr{H}_{7} \oplus \mathscr{H}_{8}$.

In the end of this section, we give an alternative proof of Theorem 2.3 in [5].

Corollary 2. Let $\mathcal{S}$ be a regular subspace of $\mathscr{H}$ with the J-selfadjoint projection $E$. Then, $E$ is uniquely written as $E=E_{1}+E_{2}$ with $E_{1}$, a J-positive projection, and $E_{2}$, a J-negative projection satisfying $E_{1} E_{2}=E_{1} E_{2}^{*}=0$.

Proof. Write $J$ and $E$ in (17) and (18), respectively. Since $Q$ is a self-adjoint operator in $\mathscr{B}\left(\mathscr{H}_{4}\right)$, there are unique positive operators $Q_{+}$and $Q_{-}$in $\mathscr{B}\left(\mathscr{H}_{4}\right)$ such that $Q=Q^{+}-Q^{-}$and $Q^{+} Q^{-}=Q^{-} Q^{+}=0$ (see $[10]$ ). Note that $0 \notin \sigma(Q), Q^{+}$, and $Q^{-}$ have closed ranges in $\mathscr{H}_{4}$, and $I_{4}=P_{\mathscr{R}\left(Q^{+}\right)}+P_{\mathscr{R}\left(Q^{-}\right)}$. Let

$$
\begin{aligned}
& E_{1}=I_{1} \oplus 0 \oplus\left(\begin{array}{cc}
P_{\mathscr{R}\left(Q^{+}\right)} & \left(Q^{+}\right)^{\dagger}\left(P_{\mathscr{R}\left(Q^{+}\right)}-\left(Q^{+}\right)^{2}\right)^{1 / 2} V \\
0 & 0
\end{array}\right) \oplus 0 \oplus 0, \\
& E_{2}=0 \oplus I_{2} \oplus\left(\begin{array}{c}
P_{\mathscr{R}\left(Q^{-}\right)}\left(Q^{-}\right)^{\dagger}\left(P_{\mathscr{R}\left(Q^{-}\right)}-\left(Q^{-}\right)^{2}\right)^{1 / 2} V \\
0
\end{array}\right) \oplus 0 \oplus 0,
\end{aligned}
$$

with respect to $\mathscr{H}=\mathscr{H}_{1} \oplus \mathscr{H}_{2} \oplus\left(\mathscr{H}_{4} \oplus \mathscr{H}_{5}\right) \oplus \mathscr{H}_{7} \oplus \mathscr{H}_{8}$. Then, $E_{1}$ and $E_{2}$ are projections, and $Q^{+} Q^{-}=Q^{-} Q^{+}=0$ implies $E=E_{1}+E_{2}$ and $E_{1} E_{2}=E_{1} E_{2}^{*}=0$.

Moreover, since

$$
\begin{aligned}
J E_{1} & =I_{1} \oplus 0 \oplus\left(\begin{array}{lr}
Q^{+} & \left(P_{\mathscr{R}\left(Q^{+}\right)}-\left(Q^{+}\right)^{2}\right)^{1 / 2} V \\
V^{*}\left(P_{\mathscr{R}\left(Q^{+}\right)}-\left(Q^{+}\right)^{2}\right)^{1 / 2} & V^{*} Q^{+\dagger}\left(P_{\mathscr{R}\left(Q^{+}\right)}-\left(Q^{+}\right)^{2}\right) V
\end{array}\right) \\
& \oplus 0 \oplus 0 \\
= & I_{1} \oplus 0 \oplus X X^{*} \oplus 0 \oplus 0 \\
& \geq 0
\end{aligned}
$$

where $X=\left(\begin{array}{cc}\left(Q^{+}\right)^{1 / 2} & 0 \\ V^{*}\left(P_{\mathscr{R}\left(Q^{+}\right)}-\left(Q^{+}\right)^{2}\right)^{1 / 2}\left(Q^{+}\right)^{\dagger(1 / 2)} & 0\end{array}\right)$, we see that $E_{1}$ is $J$-positive. Similarly, we have $J E_{2} \leq 0$, and hence, $E_{2}$ is $J$-negative. 
Now, we prove the uniqueness of $E_{1}$ and $E_{2}$. Since $J E_{1}$ and $J E_{2}$ are self-adjoint operators in $\mathscr{B}(\mathscr{H})$, we have

$$
\begin{aligned}
& \left(J E_{1}\right)\left(-J E_{2}\right)=-\left(J E_{1}\right)\left(J E_{2}\right)^{*}=-J E_{1} E_{2}^{*} J=0, \\
& \left(-J E_{2}\right)\left(J E_{1}\right)=-\left(J E_{2}\right)\left(J E_{1}\right)^{*}=-J E_{2} E_{1}^{*} J=0 .
\end{aligned}
$$

Noting that $J E=J E_{1}-\left(-J E_{2}\right)$, where $J E_{1}$ and $-J E_{2}$ are the positive operators in $\mathscr{B}(\mathscr{H})$, we see that $J E_{1}$ and $-J E_{2}$ are the positive part and the negative part of the self-adjoint operator $J E$, respectively. So $J E_{1}$ and $-J E_{2}$, and hence, $E_{1}$ and $E_{2}$ are unique.

\section{J-Self-Adjoint Projections for the Pseudoregular Subspaces}

In this section, we study the pseudoregular subspaces of $\mathscr{H}$. If $\mathcal{S}$ is a pseudoregular subspace of $\mathscr{H}$ and $\mathscr{L}$ is a complement of $\mathcal{S}^{0}$ in $\mathcal{S}$, we present a block-operator matrix representation of the $J$-self-adjoint projection onto $\mathscr{L}$.

Lemma 2. Let $\mathscr{H}$ be a Krein space with fundamental symmetry $J$, and let $\mathcal{S}$ be a closed subspace of $\mathscr{H}$. Then, the following statements are equivalent:

(a) $\mathcal{S}$ is pseudoregular

(b) There exists a regular subspace $\mathscr{M}$ such that $\mathcal{S}=\mathcal{S}^{0}[\dot{+}] \mathscr{M}$, where $[\dot{+}]$ denotes the direct $[\cdot, \cdot]$-orthogonal sum

(c) If $\mathcal{S}=\mathcal{S}^{0} \dot{+} \mathscr{T}$, then $\mathscr{T}$ is regular (d) $\mathcal{S} \ominus \mathcal{S}^{0}$ is regular

(e) The operator $Q$ in (5) is invertible

Proof. Due to Proposition 4.1 in [4], $(a) \Longleftrightarrow(b) \Longleftrightarrow(c)$.

$(c) \Longrightarrow(d)$ : since $\mathcal{S}=\mathcal{S}^{0} \dot{+}\left(\mathcal{S} \ominus \mathcal{S}^{0}\right)$, this is immediate.

$(d) \Longrightarrow(b): \quad$ since $\quad \mathcal{S} \ominus \mathcal{S}^{0}=\mathscr{H}_{1} \oplus \mathscr{H}_{2} \oplus \mathscr{H}_{4} \quad$ and $J\left(\mathcal{S}^{0}\right)=\mathscr{H}_{6}$,

$$
[x, y]=\langle J x, y\rangle=0,
$$

for all $x \in \mathcal{S}^{0}$ and $y \in \mathcal{S} \ominus \mathcal{S}^{0}$. Thus, $\mathcal{S}=\mathcal{S}^{0}[\dot{+}]$ $\left(\mathcal{S} \ominus \mathcal{S}^{0}\right)$, and hence, $(d) \Longrightarrow(b)$ is clear.

$(d) \Longleftrightarrow(e)$ : arguing as Theorem $2, \delta \ominus \mathcal{S}^{0}$ is regular if and only if the operator $Q$ in (5) is invertible.

If $\mathcal{S}$ be a pseudoregular subspace of $\mathscr{H}$ and $\mathscr{L}$ be a complement of $\mathcal{S}^{0}$ in $\mathcal{S}$, then by Lemma $2, \mathscr{L}$ is a regular subspace. The following theorem gives a block-operator matrix representation of the $J$-self-adjoint projection onto $\mathscr{L}$.

Theorem 3. Let $\mathscr{H}$ be a Krein space with fundamental symmetry $J$, and let $\mathcal{S}$ be a pseudoregular subspace of $\mathscr{H}$. Write J in (5) with respect to space decomposition (4). If $\mathscr{L}$ is a complement of $\mathcal{S}^{0}$ in $\mathcal{S}$, then the J-self-adjoint projection $E$ onto $\mathscr{L}$ has the operator matrix representation:

$$
E=\left(\begin{array}{cccccccc}
I_{1} & 0 & 0 & 0 & 0 & E_{16} & 0 & 0 \\
0 & I_{2} & 0 & 0 & 0 & E_{26} & 0 & 0 \\
U E_{16}^{*} & -U E_{26}^{*} & 0 & U E_{46}^{*} Q & U E_{46}^{*}\left(I_{4}-Q^{2}\right)^{1 / 2} V & U E_{16}^{*} E_{16}-U E_{26}^{*} E_{26}+U E_{46}^{*} Q E_{46} & 0 & 0 \\
0 & 0 & 0 & I_{4} & Q^{-1}\left(I_{4}-Q^{2}\right)^{1 / 2} V & 0 & 0 \\
0 & 0 & 0 & 0 & 0 & E_{46} & 0 & 0 \\
0 & 0 & 0 & 0 & 0 & 0 & 0 & 0 \\
0 & 0 & 0 & 0 & 0 & 0 & 0 & 0 \\
0 & 0 & 0 & 0 & 0 & 0 & 0 & 0
\end{array}\right),
$$

with respect to the space decomposition $\mathscr{H}=\oplus_{i=1}^{8} \mathscr{H}_{i}$, where $E_{i 6} \in \mathscr{B}\left(\mathscr{H}_{6}, \mathscr{H}_{i}\right), i=1,2,4$.

Proof. If $\mathscr{L}$ is a complement of $\mathcal{S}^{0}$ in $\mathcal{S}$, then $\mathscr{L}$ is regular. Suppose that $E$ is the $J$-self-adjoint projection onto $\mathscr{L}$. Since $\mathscr{L} \subseteq \mathcal{S}$, we have $P_{\mathcal{S}^{\perp}}=P_{\mathcal{S}^{\perp}} P_{\mathscr{L}^{\perp}}$, and hence,

$$
P_{\mathcal{S}^{\perp}} E=\left(P_{\mathcal{S}^{\perp}} P_{\mathscr{L}^{\perp}}\right) E=P_{\mathcal{S}^{\perp}}\left(P_{\mathscr{L}^{\perp}} E\right)=P_{\mathcal{S}^{\perp}} 0=0 .
$$

Moreover, since $\delta^{0} \subseteq \mathcal{S}^{[\perp]} \subseteq \mathscr{L}^{[\perp]}=\mathscr{N}(E)$, we get $\left.E\right|_{\mathcal{S}^{0}}=0$. Noting that $\oplus_{i=5}^{8} \mathscr{H}_{i}=\mathcal{S}^{\perp}$ and $\mathscr{H}_{3}=\mathcal{S}^{0}$, then $E$ has the operator matrix representation:

$$
E=\left(\begin{array}{cccccccc}
E_{11} & E_{12} & 0 & E_{14} & E_{15} & E_{16} & E_{17} & E_{18} \\
E_{21} & E_{22} & 0 & E_{24} & E_{25} & E_{26} & E_{27} & E_{28} \\
E_{31} & E_{32} & 0 & E_{34} & E_{35} & E_{36} & E_{37} & E_{38} \\
E_{41} & E_{42} & 0 & E_{44} & E_{45} & E_{46} & E_{47} & E_{48} \\
0 & 0 & 0 & 0 & 0 & 0 & 0 & 0 \\
0 & 0 & 0 & 0 & 0 & 0 & 0 & 0 \\
0 & 0 & 0 & 0 & 0 & 0 & 0 & 0 \\
0 & 0 & 0 & 0 & 0 & 0 & 0 & 0
\end{array}\right),
$$


with respect to the space decomposition $\mathscr{H}=\oplus_{i=1}^{8} \mathscr{H}_{i}$, where $E_{i j} \in \mathscr{B}\left(\mathscr{H}_{j}, \mathscr{H}_{i}\right)$.

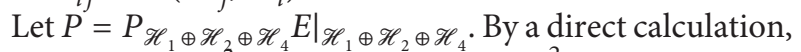

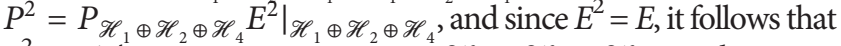
$P^{2}=P$. Thus, $P$ is a projection on $\mathscr{H}_{1} \oplus \mathscr{H}_{2} \oplus \mathscr{H}_{4}$. Furthermore, since $\mathscr{H}_{1} \oplus \mathscr{H}_{2} \oplus \mathscr{H}_{4}=\mathcal{S} \ominus \mathcal{S}^{0}$ and $\mathcal{S}^{0} \subseteq \mathscr{N}\left(P_{\mathcal{\delta} \ominus \delta^{0}}\right) \cap \mathcal{N}(E)$, we have

$$
\begin{gathered}
\mathscr{R}(P)=P_{\mathcal{S} \ominus \mathcal{S}^{0}} E\left(\mathcal{S} \ominus \mathcal{S}^{0}\right)=P_{\mathcal{S} \ominus \mathcal{S}^{0}} E(\mathcal{S})=P_{\mathcal{S} \ominus \mathcal{\delta}^{0}}(\mathscr{L}) \\
=P_{\delta \ominus \mathcal{S}^{0}}(\mathcal{S})=\mathcal{S} \ominus \mathcal{S}^{0}=\mathscr{H}_{1} \oplus \mathscr{H}_{2} \oplus \mathscr{H}_{4} .
\end{gathered}
$$

So $P$ is the identity on $\mathscr{H}_{1} \oplus \mathscr{H}_{2} \oplus \mathscr{H}_{4}$, and hence,

$$
E=\left(\begin{array}{cccccccc}
I_{1} & 0 & 0 & 0 & E_{15} & E_{16} & E_{17} & E_{18} \\
0 & I_{2} & 0 & 0 & E_{25} & E_{26} & E_{27} & E_{28} \\
E_{31} & E_{32} & 0 & E_{34} & E_{35} & E_{36} & E_{37} & E_{38} \\
0 & 0 & 0 & I_{4} & E_{45} & E_{46} & E_{47} & E_{48} \\
0 & 0 & 0 & 0 & 0 & 0 & 0 & 0 \\
0 & 0 & 0 & 0 & 0 & 0 & 0 & 0 \\
0 & 0 & 0 & 0 & 0 & 0 & 0 & 0 \\
0 & 0 & 0 & 0 & 0 & 0 & 0 & 0
\end{array}\right) .
$$

Then,

$$
J E=\left(\begin{array}{cccccccc}
I_{1} & 0 & 0 & 0 & E_{15} & E_{16} & E_{17} & E_{18} \\
0 & -I_{2} & 0 & 0 & -E_{25} & -E_{26} & -E_{27} & -E_{28} \\
0 & 0 & 0 & 0 & 0 & 0 & 0 & 0 \\
0 & 0 & 0 & Q & Q E_{45} & Q E_{46} & Q E_{47} & Q E_{48} \\
0 & 0 & 0 & X & X E_{45} & X E_{46} & X E_{47} & X E_{48} \\
U^{*} E_{31} & U^{*} E_{32} & 0 & U^{*} E_{34} & U^{*} E_{35} & U^{*} E_{36} & U^{*} E_{37} & U^{*} E_{38} \\
0 & 0 & 0 & 0 & 0 & 0 & 0 & 0 \\
0 & 0 & 0 & 0 & 0 & 0 & 0 & 0
\end{array}\right),
$$

where $X=V^{*}\left(I_{4}-Q^{2}\right)^{1 / 2}$, and since $J E$ is self-adjoint, we have

$$
\left\{\begin{array}{l}
E_{15}=0, U^{*} E_{31}=E_{16}^{*}, E_{17}=0, E_{18}=0, \\
E_{25}=0, U^{*} E_{32}=-E_{26}^{*}, E_{27}=0, E_{28}=0, \\
Q E_{45}=\left(I_{4}-Q^{2}\right)^{1 / 2} V, U^{*} E_{34}=E_{46}^{*} Q, Q E_{47}=0, Q E_{48}=0, \\
U^{*} E_{35}=E_{46}^{*}\left(I_{4}-Q^{2}\right)^{1 / 2} V, U^{*} E_{37}=0, U^{*} E_{38}=0 .
\end{array}\right.
$$

Since $U$ is an isometric isomorphism from $\mathscr{H}_{6}$ onto $\mathscr{H}_{3}$ and $0 \notin \sigma(Q)$, it also follows that

$$
\left\{\begin{array}{l}
E_{31}=U E_{16}^{*}, \\
E_{32}=-U E_{26}^{*}, \\
E_{45}=Q^{-1}\left(I_{4}-Q^{2}\right)^{1 / 2} V, E_{34}=U E_{46}^{*} Q, E_{47}=0, E_{48}=0, \\
E_{35}=U E_{46}^{*}\left(I_{4}-Q^{2}\right)^{1 / 2} V, E_{37}=0, E_{38}=0 .
\end{array}\right.
$$

Now, we see that

$$
E=\left(\begin{array}{cccccccc}
I_{1} & 0 & 0 & 0 & 0 & E_{16} & 0 & 0 \\
0 & I_{2} & 0 & 0 & 0 & E_{26} & 0 & 0 \\
U E_{16}^{*} & -U E_{26}^{*} & 0 & U E_{46}^{*} Q & U E_{46}^{*}\left(I_{4}-Q^{2}\right)^{1 / 2} V & E_{36} & 0 & 0 \\
0 & 0 & 0 & I_{4} & Q^{-1}\left(I_{4}-Q^{2}\right)^{1 / 2} V & E_{46} & 0 & 0 \\
0 & 0 & 0 & 0 & 0 & 0 & 0 & 0 \\
0 & 0 & 0 & 0 & 0 & 0 & 0 & 0 \\
0 & 0 & 0 & 0 & 0 & 0 & 0 & 0 \\
0 & 0 & 0 & 0 & 0 & 0 & 0 & 0
\end{array}\right) .
$$


Moreover, since $E^{2}=E$, we have

$$
E_{36}=U E_{16}^{*} E_{16}-U E_{26}^{*} E_{26}+U E_{46}^{*} Q E_{46} .
$$

Thus, $E$ has the asserted operator matrix.

Corollary 3. Let $\mathscr{H}$ be a Krein space with fundamental symmetry $J$, and let $\mathcal{S}$ be a pseudoregular subspace of $\mathscr{H}$. Write J in (5) with respect to space decomposition (4). Then, the J-self-adjoint projection E onto $\mathcal{S} \ominus \mathcal{S}^{0}$ has the operator matrix representation:

$$
E=I_{1} \oplus I_{2} \oplus 0 \oplus\left(\begin{array}{cc}
I_{4} & Q^{-1}\left(I_{4}-Q^{2}\right)^{1 / 2} V \\
0 & 0
\end{array}\right) \oplus 0 \oplus 0 \oplus 0,
$$

with respect to space decomposition (4).

Proof. By Theorem 3, the $J$-self-adjoint projection $E$ onto $\mathcal{S} \ominus \mathcal{S}^{0}$ has operator matrix representation (33) with respect to space decomposition (4). Moreover, since $P_{\delta^{0}}=0 \oplus 0 \oplus I_{3} \oplus$ $0 \oplus 0 \oplus 0 \oplus 0 \oplus 0$, the equation $P_{\mathcal{S}^{0}} E=0$ implies

$$
\left(\begin{array}{ccccc}
0 & 0 & 0 & 0 & 0 \\
0 & 0 & 0 & 0 & 0 \\
U E_{16}^{*} & -U E_{26}^{*} & 0 & U E_{46}^{*} Q & U E_{46}^{*}\left(I_{4}-Q^{2}\right)^{1 / 2} V \\
0 & 0 & 0 & 0 & 0 \\
0 & 0 & 0 & 0 & 0 \\
0 & 0 & 0 & 0 & 0 \\
0 & 0 & 0 & 0 & 0 \\
0 & 0 & 0 & 0 & 0
\end{array}\right.
$$

$\left.\begin{array}{ccc}0 & 0 & 0 \\ 0 & 0 & 0 \\ U E_{16}^{*} E_{16}-U E_{26}^{*} E_{26}+U E_{46}^{*} Q E_{46} & 0 & 0 \\ 0 & 0 & 0 \\ 0 & 0 & 0 \\ 0 & 0 & 0 \\ 0 & 0 & 0 \\ 0 & 0 & 0\end{array}\right)=0$.

It follows that $U E_{16}^{*}=0,-U E_{26}^{*}=0$, and $U E_{46}^{*} Q=0$. Noting that $U$ is an isometric isomorphism from $\mathscr{H}_{6}$ onto $\mathscr{H}_{3}$ and $Q$ is a self-adjoint contraction with dense range in $\mathscr{H}_{4}$, we get that $E_{16}=0, E_{26}=0$, and $E_{46}=0$. Thus, $E$ has the asserted operator matrix.

\section{Data Availability}

No data were used to support this study.

\section{Conflicts of Interest}

The authors declare that they have no conflicts of interest.

\section{Acknowledgments}

This work was supported by the NSF of China (No. 11601339) and Natural Science Foundation of Jiangsu Province of China (No. BK20171421).

\section{References}

[1] T. Ando, Linear Operators on Krein Spaces, Lecture Note, Hokkaido University, Sapporo, Japan, 1979.

[2] T. Y. Azizov and I. S. Iokhvidov, Linear Operators in Spaces with an Indefinite Metric, John Wiley \& Sons, Chichester, UK, 1989.

[3] J. Bognár, Indefinite Inner Product Spaces, Springer-Verlag, Berlin, Germany, 1974.

[4] A. Maestripieri and F. Martínez Pería, "Normal projections in Krein spaces," Integral Equations and Operator Theory, vol. 76, no. 3, pp. 357-380, 2013.

[5] T. Ando, "Projections in Krein spaces," Linear Algebra and Its Applications, vol. 431, no. 12, pp. 2346-2358, 2009.
[6] S. Hassi and K. Nordström, "On projections in a space with an indefinite metric," Linear Algebra and Its Applications, vol. 208-209, pp. 401-417, 1994.

[7] A. Gheondea, "On the geometry of pseudo-regular subspaces of a Krein space," in Spectral Theory of Linear Operators and Related Topics, pp. 141-156, Birkhäuser, Boston, MA, USA, 1984.

[8] J. I. Giribet, A. Maestripieri, and F. Martínez Pería, "Optimal normal projections in Krein spaces," Linear Algebra and Its Applications, vol. 490, pp. 77-101, 2016.

[9] H. K. Du, "Operator matrix forms of positive operator matrices," Chinese Quarterly Journal of Mathematics, vol. 7, pp. 9-11, 1992, in Chinese.

[10] J. B. Conway, A Course in Functional Analysis, Springer, New York, NY, USA, 2003. 